Juliane Stiller*, Timo Gnadt, Matteo Romanello und Klaus Thoden

\title{
Anforderungen ermitteln, Lösungen evaluieren und Erfolge messen - Begleitforschung in DARIAH-DE
}

DOI 10.1515/bfp-2016-0025

Zusammenfassung: DARIAH-DE unterstützt Geistes- und Kulturwissenschaftler mit Digital-Humanities-Tools und fachwissenschaftlichen Diensten in den Bereichen Forschung und Lehre. Der nachfolgende Artikel beschreibt die Arbeit und die Ergebnisse der wissenschaftlichen Begleitforschung innerhalb DARIAH-DE. Die Wissenschaftliche Begleitforschung hat die Aufgabe, Kriterien zu entwickeln, mit denen eine Bewertung der im Projekt entwickelten Komponenten vorgenommen werden kann. Dies geschieht in der Aufbauphase des Projektes, um erfolgreiche Impulse für eine vielversprechende Projektentwicklung geben zu können. Die Wissenschaftliche Begleitforschung gibt auch Anstöße für die erfolgreiche Überführung in den Produktivbetrieb. Grundlegend für die Arbeit ist dabei die Auseinandersetzung mit dem geisteswissenschaftlichen Forschungsablauf und den Bedürfnissen der Fachwissenschaftler. Dazu zählt auch die Entwicklung bedienbarer und nutzerfreundlicher Werkzeuge und Infrastrukturkomponenten.

Schlüsselwörter: Forschungsinfrastruktur; Begleitforschung; Evaluation; Usability; Nutzeranforderungen

\section{Identify Needs, Evaluate Solutions and Measuring Success - Accompanying Research in DARIAH-DE}

Abstract: DARIAH-DE supports humanities and cultural scholars with Digital Humanities tools and professional services in the fields of scientific research and teaching. The following article deals with the work and the results of accompanying scientific research in DARIAH-DE. The purpose of this work is to develop appropriate criteria to evaluate and assess the development of digital tools and services. This happens in the construction phase of the project in order to generate successful incentives for a promising project development. The accompanying scientific research formulates recommendations for the successful transfer of DARIAH-DE in the operational phase. The basis

*Kontaktperson: Dr. Juliane Stiller, jstiller@mpiwg-berlin.mpg.de Dipl.-Inform.Timo Gnadt, gnadt@sub.uni-goettingen.de Dr. Matteo Romanello, matteo.romanello@dainst.de M.A. Klaus Thoden, kthoden@mpiwg-berlin.mpg.de for this is the analysis of the scholarly research cycle and the needs of the scholars. This includes the development of user-friendly tools and their interfaces.

Keywords: Research infrastructure; accompanying research; research evaluation; usability; user needs

\section{Begleitforschung in DARIAH-DE}

In diesem Artikel stellen wir die Wissenschaftliche Begleitforschung und deren Arbeiten und Ergebnisse innerhalb von DARIAH-DE vor. Dieses erste Cluster von DARIAH-DE soll clusterübergreifend Forschung betreiben und Kriterien entwickeln, anhand derer die Entwicklungs- und Integrationsarbeit von DARIAH-DE richtungsgebend bestimmt werden kann.

Eine Begleitforschung ist dann sinnvoll, wenn auf Innovationen ausgerichtete Projekte in ihrer Aufbauphase empirisch untersucht werden sollen. Sie überprüft die „Umsetzung und Zielerreichung“" eines Projektes und gewinnt Erkenntnisse, „um die Wirksamkeit und den Nutzen [eines Projektes] abzuschätzen“. ${ }^{2}$ Die Begleitforschung hat die Aufgabe, passende Kriterien zu entwickeln, anhand derer das betrachtete Projekt evaluiert und die Ergebnisse vereinheitlicht werden können. Parameter wie Wirksamkeit und Nutzen werden dabei in messbare Kategorien überführt, um eine standardisierte Bewertung des Projekts und perspektivisch auch zukünftiger Projekte vorzunehmen. Calhoun (2002) gibt außerdem an, dass ein ,evaluation research“3 ${ }^{\text {* }}$ vor allem dann sinnvoll ist, wenn eine Messung an gut definierten Indikatoren (wie z. B. Profit) nicht gegeben ist. Da DARIAH-DE gerade nicht zum Ziel hat, Profit zu erwirtschaften, müssen andere Faktoren herangezogen werden, um eine Bilanz zu ziehen.

Begleitforschung dient nicht dazu, den laufenden Betrieb und die Aktivitäten innerhalb eines Projektes zu un-

1 Diaz-Bone und Weischer (2015) s.v. Begleitforschung.

2 Schubert und Klein (2011) s.v. Begleitforschung.

3 Es gibt keine wortwörtliche Übersetzung des Begriffes „Begleitforschung "ins Englische, jedoch sind nach Endruweit und Trommsdorff (2002) (s.v. Begleitforschung) die Begriffe Begleitforschung und Evaluation synonym. 
tersuchen: Dazu dienen andere Werkzeuge, beispielsweise Integriertes Monitoring, wie es für die Forschungsinfrastruktur TextGrid ${ }^{4}$ entwickelt wurde. ${ }^{5}$ Es tun sich dabei aber Parallelen auf, da auch in einem solchen MonitoringVerfahren Prozesse begleitet und auf ihren Erfolg hin überprüft werden. Die Begleitforschung ist vielmehr vorher, in der Aufbauphase angesiedelt, um hier die richtigen Anstöße für einen erfolgreichen Produktivbetrieb zu liefern und Kriterien zur späteren Bewertung festzulegen.

Die Aufbauphase von DARIAH-DE wird durch eine evaluative Forschung begleitet, weil die Entwicklung einer digitalen Forschungsumgebung für die Geistes- und Kulturwissenschaften mit einem tiefgreifenden Wandel einhergeht: die Transformation eines größtenteils analogen Forschungsprozesses in eine Arbeit mit vornehmlich digitalen Objekten, der Anwendung computergestützter Methoden in der Forschung und der Verarbeitung und Interpretation der dadurch entstehenden Datenmengen.

Eine an dieser Stelle auftretende Frage ist, mit welchen Erwartungen Wissenschaftler an eine digitale Forschungsinfrastruktur herantreten. Welche Bedürfnisse haben sie, falls sie ihre Arbeitsweise entsprechend anpassen wollen oder müssen? Und was für Faktoren spielen eine Rolle, damit sie die Benutzung der Infrastruktur als nützlich und effektiv empfinden?

Während der zweiten Förderphase von DARIAH-DE wurden dazu in der Begleitforschung zwei Bereiche genauer unter die Lupe genommen: Nutzer- und Bedienfreundlichkeit (Usability) von Forschungsumgebungen und Tools sowie die Erhebung von Erfolgskriterien. Hinsichtlich der Usability wurde im Rahmen der Begleitforschung die Gebrauchstauglichkeit der Infrastruktur untersucht: Was kann DARIAH-DE den Benutzern bieten und wo gibt es Alleinstellungsmerkmale? Dazu wurde durch eine Umfrage eine Bestandsanalyse der derzeit in der Forschung eingesetzten Software durchgeführt. ${ }^{6}$ Dabei konnten Lücken und Brüche im Arbeitsprozess identifiziert werden: Arbeitsschritte, für die es kein geeignetes Werkzeug gibt und fehlende Übergänge von Software zu Software, wo Daten zunächst konvertiert werden müssen. Solche Erhebungen helfen, die weitere Entwicklung der Infrastruktur zu steuern, um einen digitalen Forschungsalltag zu ermöglichen. Weil es wichtig ist, dass die unterschiedlichen Komponenten einer virtuellen Forschungsumgebung einen Eindruck der Zusammengehörigkeit vermitteln, wurden zusätzlich Richtlinien für neu zu entwickelnde Software erstellt. Dies betrifft aus Nutzerperspektive vor allem die Gestaltung der Benutzeroberfläche und die einheitliche Benennung der verfügbaren Befehle. Diese Richtlinien sollten Entwickler so früh wie möglich vorliegen, damit sie in den Prozess der Softwareentwicklung integriert werden können.

Die Erhebung von Erfolgskriterien dient der Aufstellung von Maßstäben, anhand derer der Erfolg einer digitalen Forschungsinfrastruktur gemessen und bewertet werden kann. Mit der Aufstellung solcher Erfolgskriterien werden Indikatoren geschaffen, die idealerweise auch für andere, ähnliche und zukünftige Forschungsinfrastrukturprojekte nützlich sind. Ein wichtiger Aspekt bei dieser Evaluation ist die Rücksichtnahme auf die verschiedenen Interessengruppen (Stakeholder). Am Aufbau und späteren Regelbetrieb sind mehrere Gruppen beteiligt: Anwender (also Geistes- und Kulturwissenschaftler und entsprechende Fachgesellschaften), Entwickler, Förderinstitutionen und Dienstanbieter (z. B. Bibliotheken). Diese verschiedenen Stakeholder haben unterschiedliche Prioritäten bezüglich der Entwicklung. So kann es für Anwender wichtig sein, dass gewünschte neue Features schnell umgesetzt werden, während es für Entwickler eher darum geht, ein stabiles System ohne ständige Änderungen zu betreiben. An solchen Stellen müssen Kompromisse eingegangen werden, um eine hohe Akzeptanz auf allen Seiten zu erreichen und den Anforderungen und Erwartungen gerecht zu werden.

Als Grundlage für die Arbeit von Cluster 1 wurde zunächst ein Modell des geistes- und kulturwissenschaftlichen Forschungskreislaufes entwickelt, um die unterschiedlichen Arbeitsschritte zu spezifizieren und damit explizit und greifbar zu machen. Dabei wurde darauf geachtet, sich auf eine einheitliche Terminologie zu verständigen, um die Kommunikation und Verständigung unter den verschiedenen an der Entwicklung beteiligten Gruppen zu erleichtern. Der Aufbau einer digitalen Forschungsinfrastruktur ist ein höchst interdisziplinäres Unternehmen und das Finden einer gemeinsamen Terminologie hilft dabei, ein Verständnis für die vielen unterschiedlichen Komponenten zu entwickeln.

Die Entwicklung des Forschungskreislaufes wird in Abschnitt 2 näher beschrieben. Abschnitt 3 erläutert, wie digitale Tools nutzerfreundlich gestaltet werden können und im letzten Abschnitt 4 geht es um die Erstellung und Bewertung von Faktoren und Kriterien für Erfolg und Impact.

\footnotetext{
4 https://textgrid.de/.

5 Vgl. Kaden und Palfner (2013).

6 Stiller et al. (2015).
} 


\section{Anforderungen anhand des Forschungskreislaufs analysieren}

Um Erwartungen der Anwender an die Forschungsinfrastruktur besser strukturieren und einordnen $\mathrm{zu}$ können, wurde der geisteswissenschaftliche Arbeitsprozess in definierbare Konzepte segmentiert, die in einer Modellierung dieser Domäne resultierte. Anhand eines solchen Modells können den Phasen wiederum Computerprogramme zugeordnet werden, welche die in einer Phase erforderlichen Arbeitsschritte besonders gut umsetzen. Es fand dazu eine Erhebung statt, die $2015^{7}$ vorläufig dokumentiert wurde. Eine abschließende Analyse ist für das Ende der zweiten Förderphase vorgesehen.

Ausgehend von einem prototypischen Forschungskreislauf wurde ein auf die geistes- und kulturwissenschaftliche Arbeit zugeschnittener Zyklus entwickelt, der sowohl analoge als auch digitale Arbeitsschritte unterstützt. Zunächst wurde dieser Zyklus mit bestehenden, hinlänglich bekannten Modellen verglichen (Abb.1), darunter das Scholarly Domain Model $^{8}$ und NeMO, ${ }^{9}$ welche die in den letzten Jahren entstandenen Ansätze wie TaDi$\mathrm{RAH}^{10}$ oder DiRT ${ }^{11}$ bündeln. Auffällige Differenzen fanden sich zum Beispiel bei den eher technisch ausgerichteten TaDiRAH-Kategorien und den eher konzeptionell verankerten Primitiven von Unsworth und dem Scholarly Domain Model. Trotz terminologischer Überschneidungen sind die Abgrenzungen zwischen den Begriffen und den dahinterstehenden Prozessen oft unklar. Auch wenn die Zielsetzung der Modelle unterschiedlich ist, so stimmen sie doch in ihrer Verfahrensweise überein, geisteswissenschaftliches Arbeiten in einzelne Prozesse zu zerlegen, um diese dann in einer digitalen Umgebung modellieren $\mathrm{zu}$ können.

Auf Grundlage der oben erwähnten Erhebung, in der Tools für die verschiedenen Prozesse des geistes- und kulturwissenschaftlichen Forschungskreislaufs abgefragt wurden, konnten folgende generische Phasen identifiziert werden: Planung und Konzeption, Recherche, Konsolidierung der Forschungsfrage, Interpretation, Publikation. Betrachtet man den Forschungsprozess als eine schrittweise Datenverarbeitung, kommt man nicht umhin, auch die entsprechende Kette von Computerprogrammen und vor allem deren Ein- und Ausgabeformate zu untersuchen. Dafür

7 Stiller et al. (2015).

8 Vgl. Gradmann und Hennicke (2012) und Unsworth (2000).

9 http://nedimah.dcu.gr/index.php?p=home.

10 Borek et al. (2014).

$11 \mathrm{http}: / /$ dirtdirectory.org/. wurde das erstellte Phasenschema durch zwei Dimensionen erweitert, die zeigen, welche Zwischenergebnisse in welchen Phasen $\mathrm{zu}$ erwarten sind und in welcher Form diese die Forschungsergebnisse widerspiegeln. Diese können in einer folgenden Phase wiederverwendet werden oder auch als eigenständige Ergebnisse gesehen werden, die den klassischen Abschluss des Forschungsprozesses eine schriftliche Publikation - begleiten, beispielsweise in Form von Bibliographien oder Datenbanken (Abb. 2).

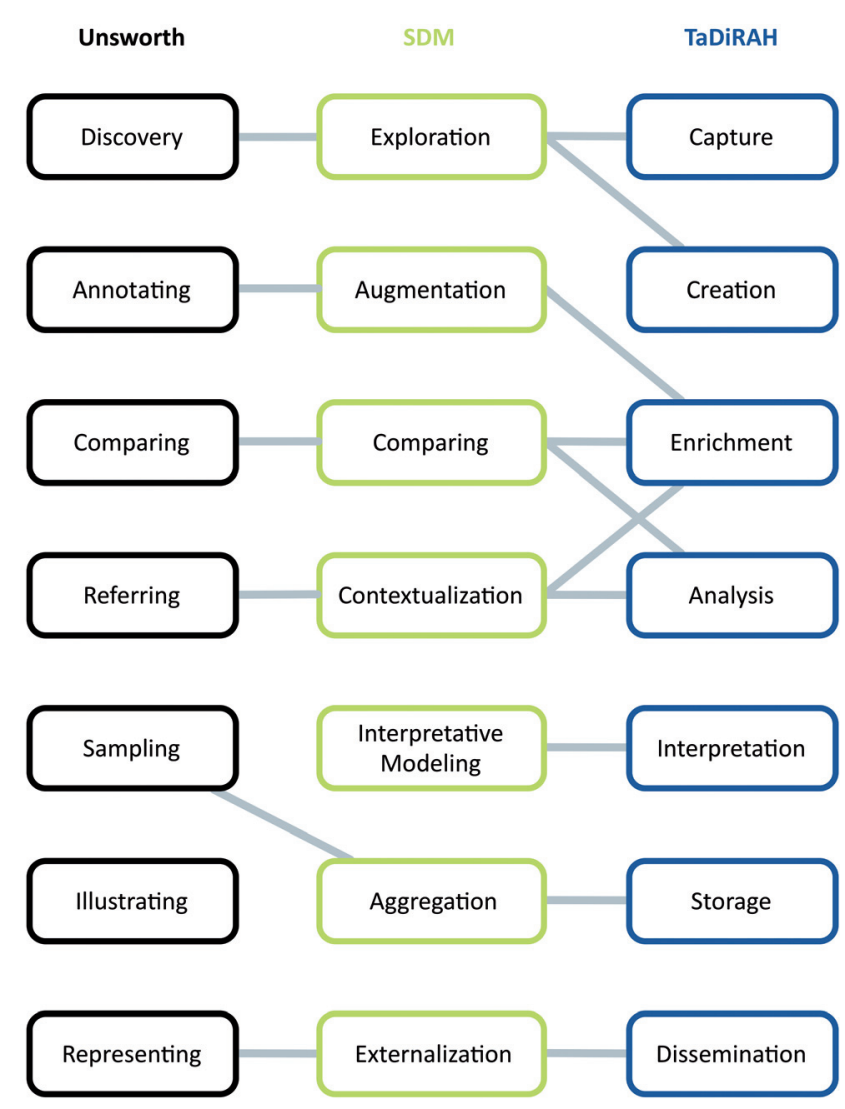

Abb. 1: Vergleich und Abbildung verschiedener Modelle und Aktivitäten des geisteswissenschaftlichen Forschungsprozesses aufeinander

Um die Forscher besser in ihrer Arbeit unterstützen zu können, ist es daher angebracht, diese Schritte noch genauer zu betrachten und festzustellen, an welchen Stellen die erforderlichen Computerprogramme fehlen, die sich gut in den Arbeitsalltag integrieren, die eine reproduzierbare Forschung ermöglichen und die möglichst offene Standards bedienen, so dass Forschungsdaten in unterschiedlichen Kontexten wiederverwendet werden können. 


\section{Forschungsaktivitäten}

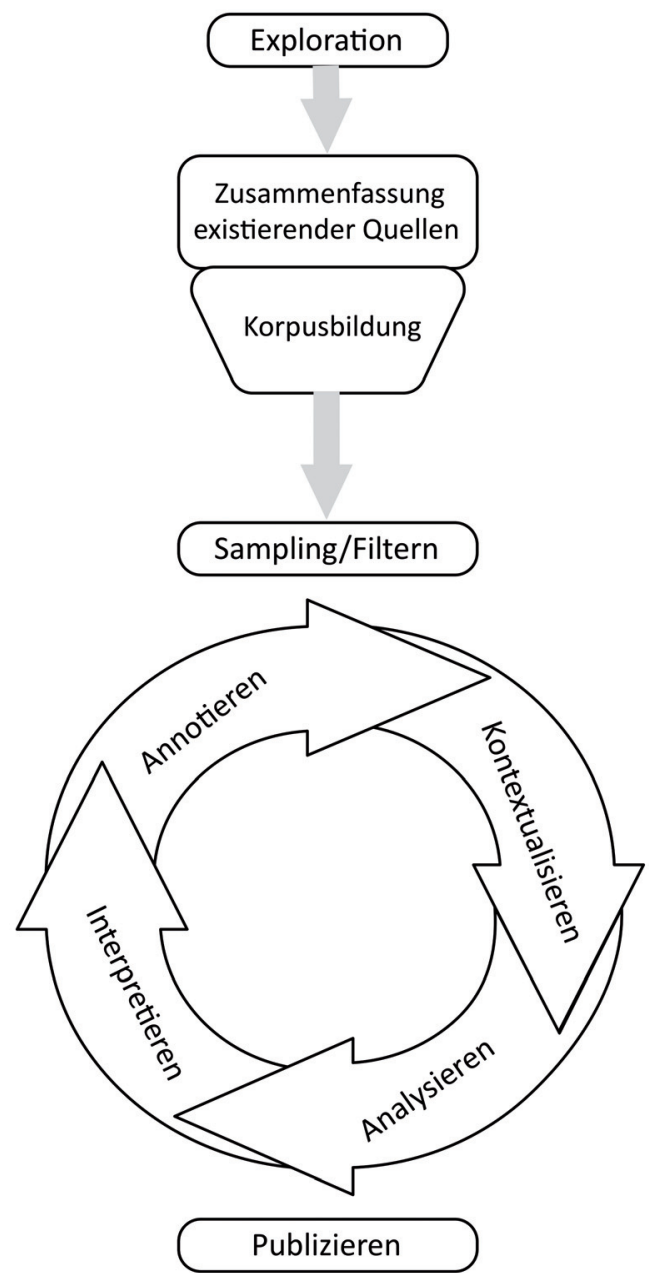

Ergebnisse

Forschungsfrage

Hypothesen

Korpus

verfeinerte

Hypothesen

neue

Hypothesen

Antworten
Wissensgenerierung

Notizen

Quellenübersicht

Bibliographien

Datenbanken

Teilkorpora

Visualisierungen

Publikation

Abb. 2: Erweiterung des Forschungsprozesses um die in den jeweiligen Phasen entstehenden Ergebnisse

\section{Digitale Tools nutzbar machen}

Usability oder auch die Nutzer- und Bedienfreundlichkeit von digitalen Tools und Forschungsumgebungen ist ein wichtiger Bestandteil für die Akzeptanz digitaler Forschungsmethoden und dient der Förderung des Einsatzes von Tools in den Geisteswissenschaften. Usability ist somit auch ein Erfolgskriterium und kann damit für eine breitere Anwendung digitaler Tools in den Geisteswissenschaften sorgen. Gibbs und Owens (2012) schreiben, dass oftmals die „potential audience [...] far larger than the targeted audience" sei und somit durch die Beachtung von Usability-Kriterien ein größerer Nutzerkreis angesprochen werden kann. Burghardt und Wolff (2015) stellen heraus, dass Erkenntnisse des Usability Engineering für eine Steigerung der Akzeptanz von DH-Tools von großer Relevanz sind. Auch wenn DH-Tools größtenteils fachspezifische Bedürf- nisse von Wissenschaftlern adressieren, so ist es doch essentiell, auch den herkömmlichen Nutzer zu berücksichtigen. Nielsen (2012) stellte in seinem Blog folgende allgemein gültigen Merkmale für nutzerfreundliche Software auf: Erlernbarkeit, Einprägsamkeit, Effizienz, Fehlertoleranz und Nutzerzufriedenheit. Inwieweit diese Merkmale bei Tools und Diensten für Kultur- und Geisteswissenschaftler in Betracht gezogen werden können, ist auch eine Frage, der sich die Begleitforschung angenommen hat. Für Cluster 1 bestand somit die Herausforderung darin, verschiedenen Nutzern gerecht zu werden und deren Bedürfnisse und Erwartungen zu identifizieren. Da DARIAH eine Infrastruktur mit vielen verschiedenen Tools ist, wurde ein Styleguide entwickelt, der darauf abzielt, die Nutzbarkeit und den Wiedererkennungswert innerhalb DARIAHs zu steigern. So wurden darin Kriterien aufgestellt und mit einer Priorisierungswertung versehen, die darauf abzielen, 
dass in DARIAH integrierte Tools gewisse Mindeststandards erfüllen. Diese sind folgenden Kategorien zugeordnet: Dokumentation, Sprache der Nutzeroberfläche, Plattform, Stabilität und Datenaustausch. ${ }^{12}$

Um die Nutzbarkeit der digitalen Tools und Dienste zu verbessern, wurden mithilfe durchgeführter Umfragen Eindrücke über die Bedürfnisse und Erfahrungen der DARIAH-Zielgruppe gesammelt.

Die Personengruppen, die DARIAH mit seinen Diensten ansprechen will, reichen von Fachwissenschaftlern aus dem Bereich der Kultur- und Geisteswissenschaften über Entwickler bis zur außeruniversitären Öffentlichkeit. Dabei will DARIAH nicht nur digital-affine Zielgruppen erreichen, sondern sucht gezielt auch den Erstkontakt und strebt an, Nutzer mit unterschiedlichen Erfahrungen im digitalen Bereich langfristig zu binden. ${ }^{13}$ Anhand der Zielgruppen wurden Use Cases erstellt, die die Bedürfnisse verschiedener Nutzergruppen widerspiegeln. Die Entwicklungen innerhalb DARIAHs wurden auf ihre Nutzerfreundlichkeit hin untersucht, wie beispielsweise der Geobrowser ${ }^{14}$ und die Publish GUI, ${ }^{15}$ die eine einfache Oberfläche für das Hochladen von Forschungsdatensammlungen bietet. Doch auch externe Tools und Dienste wurden in Kooperationen geprüft. So wurde eine Expertenevaluation am LAUDATIORepositorium ${ }^{16}$ durchgeführt und durch eine heuristische Evaluation ergänzt. ${ }^{17}$ Aufgrund dieser Erfahrungen konnten folgende häufig auftretenden Usability-Probleme geisteswissenschaftlicher Software zusammengetragen werden:

1. Inkonsistenz in der Benennung, Verwendung von mehrdeutigen Vokabularien und Missachtung graphischer Konventionen,

2. Intransparenz des Systemstatus,

3. Fehlende oder schwer auffindbare Dokumentation,

4. Fehlende Strategien zur Fehlerverhütung,

5. Missachtung von Konventionen bei der Suche oder der Strukturierung von Informationen.

Auf diese häufigen Usability-Probleme aufmerksam zu machen, ist der erste Schritt sie aktiv zu vermeiden und entsprechende Evaluationen schon früh in den Entwicklungsprozess einzubauen.

12 Romanello, Stiller und Thoden (2015).

13 Göbel et al. (2015).

$14 \mathrm{http://geobrowser.de.dariah.eu/.}$

15 https://de.dariah.eu/repository.

$16 \mathrm{http}: / /$ www.laudatio-repository.org/.

17 Stiller, Thoden und Zielke (2016).

\section{Erfolge messen und Impact schaffen $^{18}$}

Digitale Forschungsinfrastrukturen in den Kultur- und Geisteswissenschaften müssen sich natürlich auch damit auseinandersetzen, welchen Mehrwert sie einerseits für die wissenschaftliche Community schaffen und wie sie andererseits die in sie investierten Mittel nutzbringend verwenden, um dies auch vor der allgemeinen Öffentlichkeit rechtfertigen zu können. Ein Forschungsziel von Cluster 1 ist es deswegen, dezidiert für die Geisteswissenschaften nutzbare Erfolgsfaktoren und Impactkriterien zu generieren. Diese sollen nicht nur allein auf der Basis quantitativer Kriterien, wie beispielsweise Nutzungszahlen, erstellt werden, sondern auch explizit unter Erarbeitung und Begutachtung qualitativer Faktoren.

Da die Verwendung der Begriffe Impact, Erfolg, Kriterien und Faktoren in der Literatur und auch in einschlägigen Diskussionen häufig synonym oder unscharf verwendet werden, soll hier kurz der Fokus der Forschung in DARIAH-DE Cluster 1 dargestellt werden.

Impact bezeichnet hiernach die Form, den Grad oder die Diversität, der durch eine Maßnahme oder ein Produkt bewirkten Änderung eines Verhaltens oder einer Einstellung einer Gruppe. Dies impliziert bereits eine gewisse Unschärfe nicht nur des Ausmaßes der Veränderung, sondern insbesondere der konkreten Bereiche, in denen diese Veränderung auftritt. Eine umfassende Spezifikation von Indikatoren, anhand derer Impact gemessen oder beurteilt werden kann, wird hierdurch stark erschwert.

Erfolg hingegen bezeichnet eine positive Resonanz auf eine Maßnahme oder ein Produkt, welche in ihrer Stärke und ihrem Ausmaß messbar ist. Die für diese Messbarkeit erforderlichen Indikatoren (z. B. Anzahl Nutzer, Klick- oder Verkaufszahlen) können meist recht gut im Voraus spezifiziert werden.

Die Unterscheidung zwischen Faktoren und Kriterien ist nach unserem Verständnis ebenso wichtig. Während Faktoren eher Eigenschaften oder Mittel zur Veränderung eines Zustands (also z. B. zur Beeinflussung von Impact oder Erfolg) beschreiben, sind mit Kriterien meist konkrete Merkmale zur Unterscheidung zwischen Zuständen, also zur Messbarkeit des Ausmaßes von Erfolg oder Impact gemeint.

Wenngleich die Themen Erfolgsmessung, Impact und Evaluation in zahlreichen Publikationen auch für den Be-

18 Dieser Abschnitt basiert auf dem im Dezember 2015 fertig gestellten Report von Gnadt et al. (2015). 
reich der Digital Humanities behandelt werden, so finden sich doch kaum Erkenntnisse zu der Frage, welche Faktoren für welche der verschiedenen Zielgruppen relevant sind und welche Kriterien angewendet werden sollten. Insbesondere wird meist offen gelassen, welche Schlüsse hieraus für die Entwicklung, Anpassung und das Angebot von digitalen Diensten gezogen werden können.

Die Analyse von Nutzeranforderungen zu einem Portal in den Kunst- und Geisteswissenschaften von Brown et al. (2006) im Kontext des RePAH-Projektes erfolgte durch Literaturanalyse, Online-Fragebögen, Server-Log-Analysen, Fokusgruppen, Interviews und Delphi Rating. Ein relevantes Ergebnis hierbei waren die Anforderungen, welche Benutzer bei der Entwicklung zusätzlicher Funktionalitäten erheben: Diese sollten „simple, adapted to their needs, did not replicate functionality available elsewhere, was not monolithic, was capable of being managed by them, and requiring no significant investment of time to understand and use“19 sein. Des Weiteren monierten Nutzer mangelnde Interoperabilität sowohl im Hinblick auf Metadaten-Harvesting von digitalen Ressourcen, als auch zwischen bibliographischen Tools und digitalen Ressourcen. Darüber hinaus verlangten die Nutzer nach mehr Möglichkeiten zum persönlichen Management der eigenen Forschungsumgebung, wie Workflow-Management, erweiterte Lesezeichenverwaltung und ein automatisches Copyright-Management. Auch Funktionalitäten zum Auffinden von Ressourcen sowie zur verteilten Suche und Filterung wurden als relevant genannt. Auf der anderen Seite fanden Brown et al. bei den Nutzern eine hohe Skepsis gegenüber der Preisgabe von Informationen bezüglich Kollaborations- und Kommunikationstools. Dies dürfte sich entgegen den Erwartungen der Autoren in der PostSnowden-Ära nicht sonderlich verbessert haben.

Das LAIRAH-Projek $\mathrm{t}^{20}$ analysierte Logdateien zur Verwendung von „DH resources“, im Wesentlichen digitale Sammlungen, und führte ergänzend Interviews mit einigen Projektverantwortlichen durch. Hierbei wurden vier relevante Bereiche identifiziert („Content“, „Users“, „Management“ und „Dissemination“), zu denen jeweils Teilaspekte für das Erreichen einer hohen Akzeptanz und Wirkung bei wissenschaftlichen Nutzern ausformuliert und in Form einer Checkliste bereitgestellt wurden. In einer Anwendungsstudie hierzu evaluierten Warwick et al. (2007) ein konkretes Projekt anhand der entwickelten Checkliste. Hierbei handelt es sich größtenteils um allgemeine Empfehlungen („clearly identify target group, establish contact and maintain it“) oder um grundlegende technische Pflichten (,retain and make available server logs"), welche die verschiedenen Aspekte von und Anforderungen an Dienste, Methoden und größtenteils auch Infrastrukturen nicht erfassen. Diese Checkliste kann somit nur stark eingeschränkt auf den Bereich der digitalen Tools und Infrastrukturen übertragen werden.

In ihrer Analyse von drei britischen Datenzentren kombinieren Beagrie und Houghton (2014) ökonomische Ansätze zur Kosten-Nutzen-Analyse mit qualitativen Methoden wie Interviews, Surveys, Fallstudien und Fokusgruppen, sowie das ebenfalls von Beagrie und Houghton entwickelte KRDS Benefits Framework. Die Zielgruppe ihres Berichtes sind hierbei sowohl Datenzentren und -provider als auch Förderer. Ihre resultierenden Empfehlungen beschränken sich ebenfalls vorwiegend auf generelle Aspekte, wie die Befürwortung des freien Zugangs zu Daten und Diensten, sowie die Realisierung von Langzeitarchivierung und Dissemination von Daten.

Innerhalb des DFG-geförderten Projekts „Erfolgskriterien für den Aufbau und nachhaltigen Betrieb von Virtuellen Forschungsumgebungen (DFG-VRE)“ wurden Erfolgskriterien anhand eines Lebensphasenmodells für Förderer, Betreiber und Nutzer von Virtuellen Forschungsumgebungen erstellt. ${ }^{21}$ Innerhalb unserer Arbeit in DARIAH-DE haben wir diese Ausarbeitung als Grundlage genommen, um den Rahmen für unsere Forschungsarbeit zu Erfolgskriterien zu setzen.

Die zwei bisher in DARIAH-DE durchgeführten Umfragen zeigen starke Ausprägungen von drei Gruppen von Faktoren, welche über alle Stakeholdergruppen in den Geisteswissenschaften als zentral für den Erfolg von digitalen Tools und Infrastrukturkomponenten angesehen werden. Bei diesen Gruppen handelt es sich zum einen um Usability-Aspekte wie Erlernbarkeit, Bedienbarkeit und Dokumentation, zum zweiten um technische Merkmale wie Stabilität, Verfügbarkeit, Schnittstellen und Infrastruktur-Anbindung und zum dritten um Community-Aspekte wie Einbeziehung von Nutzern, Sichtbarkeit, $\mathrm{Zu}$ gänglichkeit und Kollaboration.

Die erste dieser beiden Umfragen wurde im Sommer 2014 durchgeführt und zielte darauf ab, die Relevanz von Erfolgskriterien von digitalen Werkzeugen und Forschungsinfrastrukturen $\mathrm{zu}$ bewerten..$^{22}$ In allen abgefragten Bereichen spielte die Zuverlässigkeit und Verfügbarkeit der Dienste eine herausragende Rolle bei deren Bewertung. Weniger stark gewichtet wurden Kriterien wie

21 Buddenbohm et al. (2014).

22 Gnadt, Stiller und Höckendorff (2014). 


\section{Anzahl vergebener Punkte}

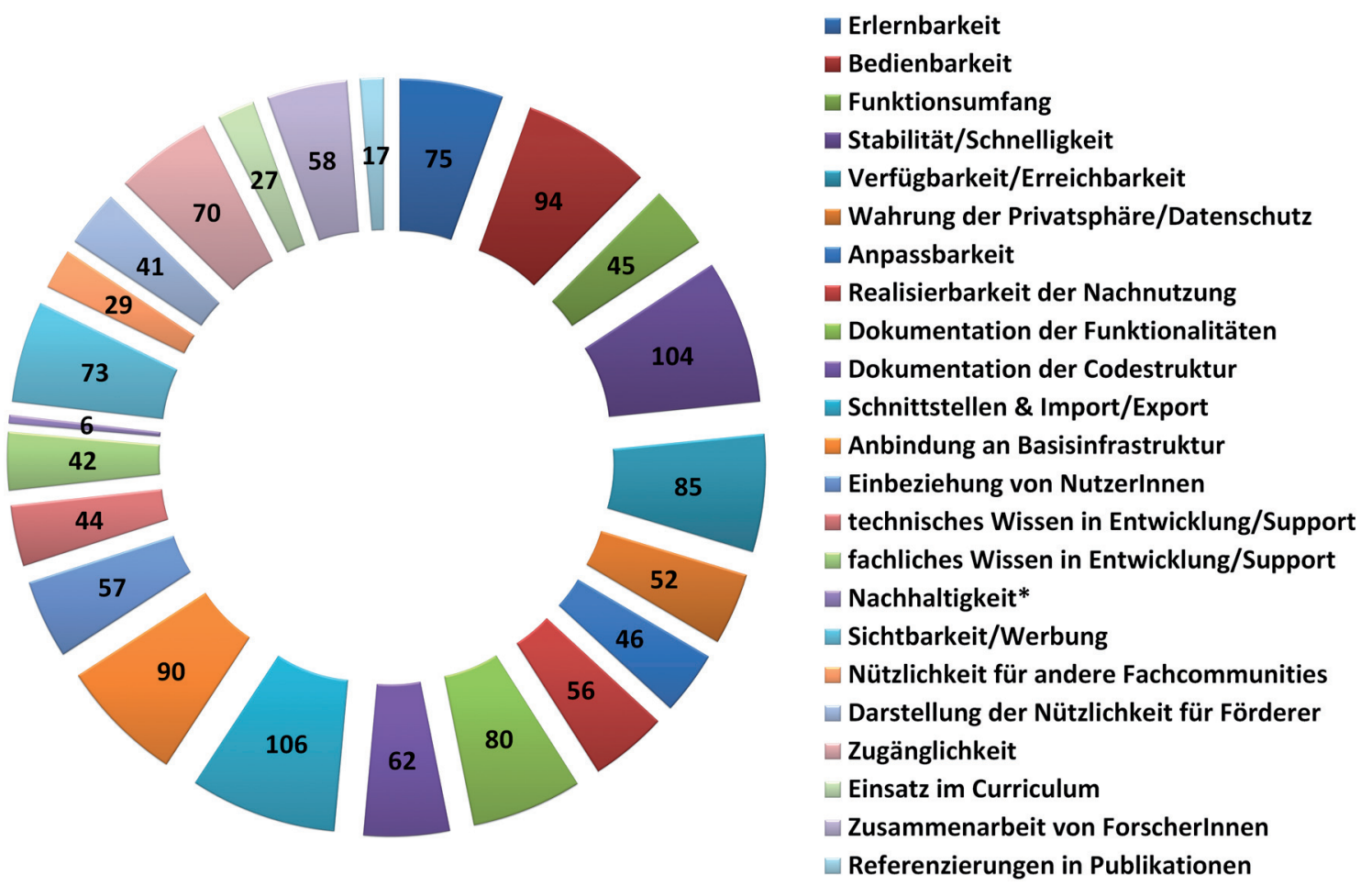

*) von 2 Nutzern manuell hinzugefügt

Abb. 3: Anzahl vergebener Punkte zur Priorisierung von Eigenschaften eines fiktiven Tools

beispielsweise die Echtzeit-Kollaboration. In den Freitextfeldern wurden häufig auch zusätzliche Kriterien benannt, welche die Zugänglichkeit und Nachhaltigkeit der Dienste betreffen.

Ähnliche Ergebnisse wurden bei der Befragung von Diensteanbietern und Diensteentwicklern ermittelt. In einem DINI-Workshop zum Thema „Digitales Arbeiten in den Geisteswissenschaften“23 wurden die Teilnehmer gebeten, sich ein fiktives DH-Tool auszudenken und dessen zu entwickelnde Eigenschaften zu priorisieren. Die gleiche Umfrage wurde in der Folge nochmals mit Diensteanbietern und Diensteentwicklern in DARIAH-DE durchgeführt. Ein Ergebnis der zusammengeführten Umfragen ist in Abb. 3 veranschaulicht.

Für die drei vorgegebenen Bereiche (1) Nutzung des Tools, (2) technische und fachliche Integration, sowie (3) Außenwirkung und Sichtbarkeit konnten die Teilnehmer in ihrer Anzahl limitierte Punkte für verschiedene Eigenschaften vergeben. Dies sollte die Probanden dazu anhalten, sich bei der Vergabe der Punkte für bestimmte Eigen-

23 https://dini.de/veranstaltungen/workshops/digitales-arbeiten-i n-den-geisteswissenschaften-ermoeglichen/. schaften ihres erdachten Tools zu entscheiden. Die jeweils wichtigsten Kriterien in den drei genannten Bereichen waren: (1) Stabilität/Schnelligkeit, (2) Schnittstellen \& Import/Export-Optionen, (3) Sichtbarkeit/Werbung. In der im Anschluss erfolgten Diskussion betonten die Teilnehmer nochmals die Wichtigkeit der Nachhaltigkeit als Kriterium für erfolgreiche Tools.

Die durchgeführten explorativen Studien mit verschiedenen Nutzergruppen und Stakeholdern von digitalen Forschungsinfrastrukturen haben gezeigt, dass sich die Faktoren für Erfolg und Impact aus Sicht dieser Gruppen nicht sehr stark voneinander unterscheiden. Die Schwierigkeit liegt eher darin, messbare Kriterien aufzustellen, mit denen qualitative Impactbereiche wie bessere Vernetzung und interdisziplinäre Zusammenarbeit bewertet werden können.

Der von Cluster 1 in tabellarischer Form erstellte „Katalog von Kriterien für Impact und Erfolg digitaler Tools und Infrastrukturkomponenten" ${ }^{24}$ bietet daher sowohl die Möglichkeit, durch Anpassung einzelner Faktoren zielgruppenspezifisch den Impact eines Tools oder Dienstes

24 Gnadt et al. (2015). 
zu beeinflussen, als auch Kriterien zur Messung des Erfolgs dieses Tools, aufgeschlüsselt nach verschiedenen Impact-Bereichen, zu bestimmen.

\section{Fazit}

Der vorliegende Artikel hat die Arbeiten der Wissenschaftlichen Begleitforschung in der zweiten Förderphase von DARIAH-DE beleuchtet. Der Fokus lag in dieser Zeit auf der Erforschung der Bedürfnisse der Fachwissenschaftler und wie auf diese in von DARIAH bereitgestellten Diensten und Services eingegangen werden kann. Nutzerfreundliche Interfaces spielen dabei eine entscheidende Rolle, um die Akzeptanz digitaler Tools und Services in den Fachcommunities zu erhöhen. Die einfache Bedienbarkeit bildet somit ein entscheidendes Erfolgskriterium für virtuelle Forschungsumgebungen und Tools. Weitere Faktoren und Kriterien für Erfolg und Impact wurden aus der Literatur und selbst durchgeführten Umfragen zielgruppenspezifisch erfasst und in einem Katalog publiziert. Die explizite Anwendung solcher Kriterien ist unerlässlich für die Entwicklung von Infrastrukturen und darauf aufbauenden virtuellen Umgebungen. ${ }^{25}$

\section{Literaturverzeichnis}

Beagrie, Neil; Houghton, John (2014): The Value and Impact of Data Sharing and Curation. A synthesis of three recent studies of UK research data centres. Verfügbar unter http://repository.jisc.ac. uk/5568/1/iDF308_-_Digital_Infrastructure_Directions_Report, Jan14_v1-04.pdf.

Borek, Luise; Dombrowski, Quinn; Munson, Matthew; Perkins, Jody; Schöch, Christof (2014): Scholarly Primitives Revisited: Towards a Practical Taxonomy of Digital Humanities Research Activities and Objects. Proceedings of the International Conference on Dublin Core and Metadata Applications, Austin, 2014, 181-183. Verfügbar unter http://dharchive.org/paper/DH2014/Paper-504 .xml.

Brown, Stephen; Ross, Robb; Gerrad, David; Greengrass, Mark; Bryson, Jared (2006): RePAH: A User Requirements Analysis for Portals in the Arts and Humanities. Leicester; Sheffield: De Montfort University Leicester und The University of Sheffield. Verfügbar unter http://repah.dmu.ac.uk/report/pdfs/RePAHReport-Co mplete.pdf.

Buddenbohm, Stefan; Enke, Harry; Hofmann, Matthias; Klar, Jochen; Neuroth, Heike; Schwiegelshohn, Uwe (2014): Erfolgskriterien für den Aufbau und nachhaltigen Betrieb Virtueller Forschungsumgebungen. DARIAH-DE Working Papers 7. Göttingen: DARIAH-

25 Wir danken Viola E. Schmitt vom Max-Planck-Institut für Wissenschaftsgeschichte für wichtiges Feedback und Korrekturen für diesen Artikel.
DE. Verfügbar unter http://webdoc.sub.gwdg.de/pub/mon/dar iah-de/dwp-2014-7.pdf.

Burghardt, Manuel; Wolff, Christian (2014): Humanist-Computer Interaction: Herausforderungen Für Die Digital Humanities aus Perspektive der Medieninformatik. In: Book of Abstracts. Workshop: „Informatik und die Digital Humanities“. Leipzig, 3.11.2014. Verfügbar unter http://dhd-wp.hab.de/files/book_of_abstracts. pdf.

Calhoun, Craig Jackson. 2002. Dictionary of the Social Sciences. New York: Oxford University Press.

Diaz-Bone, Rainer; Weischer, Christoph (2015): Methoden-Lexikon für die Sozialwissenschaften. Wiesbaden: Springer VS. Verfügbar unter http://link.springer.com/book/10.1007\%2F978-3-531-188 89-8.

Endruweit, Günter; Trommsdorff, Gisela (2002): Wörterbuch der Soziologie. Stuttgart: Lucius \& Lucius.

Gibbs, Frederick; Owens, Trevor (2012): Building Better Digital Humanities Tools: Toward broader audiences and user-centered designs. In: Digital Humanities Quarterly, 6 (2). Verfügbar unter htt p://www.digitalhumanities.org/dhq/vol/6/2/000136/000136. html.

Gnadt, Timo; Stiller, Juliane; Höckendorff, Mareike (2014): Umfrage zu Erfolgskriterien (R1.3.1). Göttingen: DARIAH-DE. Verfügbar unter https://wiki.de.dariah.eu/download/attachments/14651583/R \%201.3.1\%20-\%20Erhebung\%20einer\%20Nutzerbefragung\%2 0zu\%20Nutzererwartungen\%20und\%20-kriterien.pdf.

Gnadt, Timo; Stiller, Juliane; Thoden, Klaus; Schmitt, Viola (2015): Finale Version. Erfolgskriterien (R1.3.3.). Göttingen: DARIAH-DE. Verfügbar unter https://wiki.de.dariah.eu/download/attach ments/14651583/R133_Erfolgskriterien_Konsortium.pdf?versio $\mathrm{n}=1$ \& modificationDate=1452590465335\&api=v2.

Göbel, Mathias; Grupe, Nadja; Heise, Christian; Köhlmann, Maren; Meyer, Katharina; Neuschäfer, Markus; Schmunk, Stefan; Söring, Sibylle (2015): DARIAH-DE und TextGrid. Disseminationsstrategie inklusive Marketingkonzept sowie DARIAH-DE Open Mission Statement und Publikationsstrategie. Göttingen: DARIAH-DE. Verfügbar unter https://textgrid.de/fileadmin/TextGrid/ reports/DARIAH-TextGrid-Disseminationskonzept.pdf.

Gradmann, Stefan; Hennicke, Steffen (2012): Intermediary Research Report on DH Scholarly Primitives (MS 3). Impact Study Project. Verfügbar unter DM2E.http://dm2e.eu/files/D3.4_2.0_Researc h_Report_on_DH_Scholarly_Primitives_150402.pdf.

Kaden, Ben; Palfner, Sonja (2013): Dimensionen des Integrierten Monitoring einer digitalen Forschungsinfrastruktur und Konzept, Beurteilungsmethodik, Beurteilungskategorien und Governance-Werkzeuge. R 6.1.1 und 6.1.2. Verfügbar unter https://text grid.de/fileadmin/user_upload/TextGrid_R6.1.1undR6.1.2_inte grietes_Monitoring_und_Governance.Werkzeuge.pdf.

Nielsen, Jakob (2012): Usability 101. Introduction to usability. Verfügbar unter http://www.nngroup.com/articles/usability-101-intro duction-to-usability/.

Romanello, Matteo; Stiller, Juliane; Thoden, Klaus (2015): Usability Criteria for External Requests of Collaboration. (R. 1.2.2/R7.5). Göttingen: DARIAH-DE. Verfügbar unter https://wiki.de.dariah.e u/download/attachments/14651583/R1.2.2_Usability_Criteria_f or_External_Requests_of_Collaboration.pdf?version=1\&modific ationDate=1431088356605\&api=v2.

Schubert, Klaus; Klein, Martina (2011): Das Politiklexikon. Bonn: Dietz. Verfügbar unter http://www.bpb.de/nachschlagen/lexi ka/politiklexikon. 
Stiller, Juliane; Thoden, Klaus; Zielke, Dennis (2016): Usability in den Digital Humanities am Beispiel des LAUDATIO-Repositoriums. DHd-Tagung 2016.

Stiller, Juliane; Thoden, Klaus; Leganovic, Oona; Heise, Christian; Höckendorff, Mareike; Gnadt, Timo (2015): Nutzungsverhalten in den Digital Humanities. (R1.2.1/M 7.6). Göttingen: DARIAH-DE. Verfügbar unter https://wiki.de.dariah.eu/download/attach ments $/ 26150061 /$ Report1.2.1-final.pdf?version=6\&modification Date $=1430303390972 \&$ api $=$ v2.

Unsworth, John (2000): Scholarly Primitives: What Methods Do Humanities Researchers Have in Common, and How Might Our Tools Reflect This? Part of a symposium on Humanities Computing: formal methods, experimental practice. London: King's College. Verfügbar unter http://people.brandeis.edu/ unsworth/King s.5-00/primitives.html.

Warwick, Claire; Terras, Melissa; Galina, Isabel; Huntington, Paul; Pappa, Nikoleta (2007): Evaluating Digital Humanities Resources: The LAIRAH Project Checklist and the Internet Shakespeare Editions Project. University College London: School of Library, Archive and Informations Studies. http://elpub.scix.net/data/w orks/att/144_elpub2007.content.pdf.

Warwick, Claire; Terras, Melissa; Huntington, Paul; Pappa; Galina, Isabel (2006): The LAIRAH Project: Log Analysis of Digital Resources in the Arts and Humanities. Final Report to the Arts and Humanities Research Council. University College London: School of Library, Archive and Informations Studies. Verfügbar unter htt p://www.ucl.ac.uk/infostudies/claire-warwick/publications/LA IRAHreport.pdf.

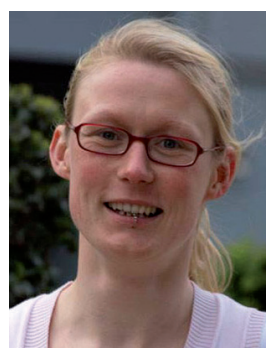

Dr. Juliane Stiller

Max-Planck-Institut für

Wissenschaftsgeschichte

Boltzmannstr. 22

D-14195 Berlin

jstiller@mpiwg-berlin.mpg.de

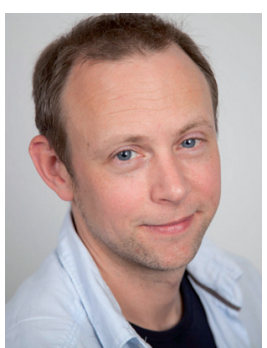

Dipl.-Inform. Timo Gnadt

Niedersächsische Staats- und

Universitätsbibliothek Göttingen

Abteilung Forschung und Entwicklung

Platz der Göttinger Sieben 1

D-37073 Göttingen

gnadt@sub.uni-goettingen.de

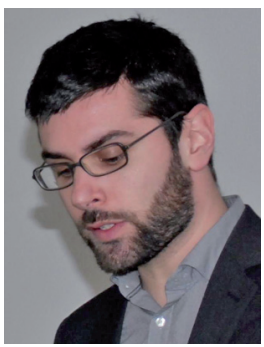

\section{Dr. Matteo Romanello}

Digital Humanities Berlin

IT-Referat

Podbielskiallee 69-71

D-14195 Berlin-Dahlem

matteo.romanello@dainst.de

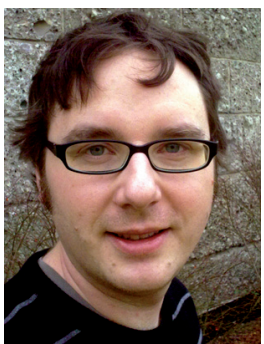

M. A. Klaus Thoden

Max-Planck-Institut für

Wissenschaftsgeschichte

Boltzmannstr. 22

D-14195 Berlin

kthoden@mpiwg-berlin.mpg.de 